\title{
Lapatinib in II linea
}

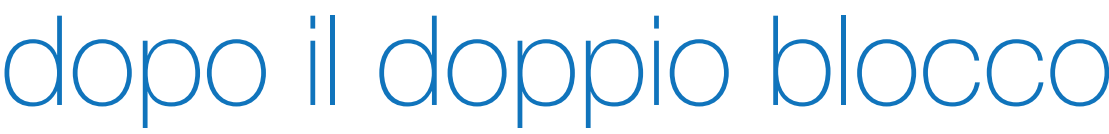

pertuzumab-trastuzumab:

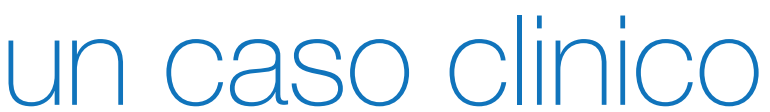

\author{
Valentina Magri, Simone Scagnoli, Gabriele Piesco, Giulia Pomati \\ Oncologia, Policlinico Umberto I, Roma
}

\section{Abstract}

Riportiamo il caso di una giovane paziente di 36 anni, con diagnosi di carcinoma della mammella multifocale, sottoposta a chemioterapia neoadiuvante, chirurgia e trattamento adiuvante.

La paziente ha presentato una precoce ripresa di malattia a livello epatico, dopo soli 7 mesi di intervallo libero. La ri-caratterizzazione istologica dopo biopsia epatica ha evidenziato una malattia HER2-positiva, per cui è stata trattata in I linea chemioterapica con doppio blocco anti-HER2. Dopo 3 cicli la malattia è progredita a livello epatico ed encefalico. Si è deciso pertanto di trattare le loca-

\section{Indirizzo per la corrispondenza:}

Valentina Magri

e-mail: magri.v@hotmail.it

Accettato: 04/07/2018 - Pubblicato online: 05/11/2018

(C) 2018 The Authors. This article is published by HPS Srl and licensed under Creative Commons Attribution-NC-ND 4.0 International (CC BY-NC-ND 4.0). Any commercial use is not permitted and is subject to Publisher's permissions. Full information is available at www.aboutpharma.com/publishing/riviste/aboutopen/ lizzazioni cerebrali con radioterapia whole-brain e iniziare una II linea con capecitabina associata a lapatinib. Questo approccio chemio-radioterapico ha permesso di controllare la malattia per 8 mesi. In seguito a ulteriore progressione (linfonodale $\mathrm{e}$ ossea) si è optato per una III linea con trastuzumab emtansine (TDM1). La malattia encefalica è rimasta stabile per altri 8 mesi, quando per progressione viscerale e scadimento delle condizioni generali, si è deciso di interrompere TDM1 e avviare le terapie di supporto.

\section{Situazione clinica iniziale}

Nel gennaio 2013, una paziente di 36 anni, in buone condizioni generali e senza comorbilità di rilievo, si sottopone a ecografia mammaria che mostra multiple nodularità a carico della mammella sinistra, con un quadro di malattia multifocale cT2 (m). Alla biopsia mammaria, l'esame istologico riscontra un carcinoma duttale infiltrante, positività ai recettori estrogenici (ER) $70 \%$, recettori progestinici $(\mathrm{PgR})<1 \%$, indice di proliferazione Ki67 11\%, HER2 1+. L'esa- 
me citologico sulla linfoadenopatia ascellare risulta positivo. In considerazione della giovane età e dello stadio di malattia cT2 (m) cN1, si decide di intraprendere una chemioterapia neoadiuvante.

Da gennaio 2013, la paziente riceve 4 cicli di chemioterapia secondo lo schema EC (epirubicina $90 \mathrm{mg} / \mathrm{m}^{2}$ e ciclofosfamide $600 \mathrm{mg} / \mathrm{m}^{2}$ ), conseguendo una minima risposta strumentale alla risonanza magnetica (RM) mammaria. Si prosegue la terapia neoadiuvante con 4 cicli di docetaxel $75 \mathrm{mg} / \mathrm{m}^{2}$, raggiungendo una risposta parziale alla RM eseguita al termine della chemioterapia.

Nel giugno 2013 si effettua una mastectomia sinistra secondo Madden. L'esame istologico definitivo depone per carcinoma lobulare infiltrante, pT1a, N1a; ER 90\%, PgR 0\%, Ki67 25\%, HER score 2+, FISH non amplificata. La paziente prosegue con radioterapia adiuvante, terminata a settembre 2013, e ormonoterapia adiuvante con tamoxifene e analogo dell'ormone di rilascio ipotalamico delle gonadotropine (LHRH).

\section{Approccio terapeutico \\ alla malattia metastatica}

Dopo un intervallo libero da malattia di 7 mesi, i controlli clinico-strumentali riscontrano una progressione strumentale a livello epatico. La tomografia computerizzata (TC) di gennaio 2014 evidenzia, infatti, multipli noduli epatici, di cui il maggiore di dimensione $107 \times 93 \times 50 \mathrm{~mm}$ al V segmento epatico (Figura 1A), oltre ad altre nodulazioni target così localizzate: due strettamente adiacenti fra loro in corrispondenza dei segmenti VII e VIII, in sede sub-glissoniana di dimensioni maggiori di 21 e 23 $\mathrm{mm}$ (Figura 1B), quattro ravvicinate nel IV segmento di cui la più grande con dimensione maggiore di $20 \mathrm{~mm}$ (Figura 1C), una lesione a margini irregolari di dimensione maggiore di $45 \mathrm{~mm}$ al V segmento (Figura 1D).

Il referto della biopsia epatica eseguita a febbraio 2014 indica un carcinoma duttale infiltrante ER 80\%, PgR 0\% e HER2 score 3.
Si decide pertanto di trattare la paziente con chemioterapia di I linea secondo lo schema pertuzumab $420 \mathrm{mg}$ giorno 1 , trastuzumab $6 \mathrm{mg} / \mathrm{kg}$ giorno 1 , paclitaxel $80 \mathrm{mg} / \mathrm{m}^{2}$ giorni $1,8,15$ da ripetersi ogni 21 giorni. Da marzo 2014 a giugno 2014 la paziente riceve 3 cicli del suddetto trattamento.

La TC total body di rivalutazione a giugno 2041 evidenzia una progressione di malattia a livello epatico, polmonare ed encefalico. Le lesioni encefaliche segnalate come target risultano così localizzate: corona radiata (dimensione maggiore $8 \mathrm{~mm}$ ) con edema perilesionale (Figura 2A), sede frontale sottocorticale (dimensione maggiore $7 \mathrm{~mm}$ ) [Figura 2B], sede cerebellare sinistra (dimensione maggiore $5 \mathrm{~mm}$ ) [Figura 2C]; la risonanza magnetica (RM) encefalica conferma il quadro di multiple lesioni encefaliche.

A luglio 2014, la paziente si sottopone a radioterapia whole-brain e inizia una terapia di II linea secondo lo schema capecitabina-lapatinib. A ottobre 2014 la TC total body di controllo mostra un quadro di risposta parziale a livello epatico ed encefalico. Le lesioni target epatiche appaiono modificate: nel V segmento epatico la maggiore passa da $107 \times 93 \mathrm{~mm}$ a $55 \times 35 \mathrm{~mm}$, mentre le restanti al VII/VIII, IV e V sono lievemente ridotte.

Dopo un intervallo libero da progressione di 8 mesi, alla TC di rivalutazione di febbraio 2015 si riscontra una ulteriore risposta parziale epatica (Figura 3) e una risposta encefalica completa (Figura 4), ma contestualmente una progressione linfonodale e ossea.

La RM conferma il quadro di risposta encefalica completa. Viene però rilevata progressione biochimica con aumento del marker Ca15.3. La paziente è quindi avviata a una terapia di III linea secondo lo schema TDM1, che le viene somministrata per 4 cicli, da luglio a settembre 2015. Alla visita di rivalutazione di ottobre 2015 si evidenziano un peggioramento delle condizioni cliniche generali e una progressione strumentale a livello osseo ed epatico, mentre alla RM encefalica persiste la risposta completa; si avvia pertanto la paziente alla sola terapia di supporto domiciliare. 

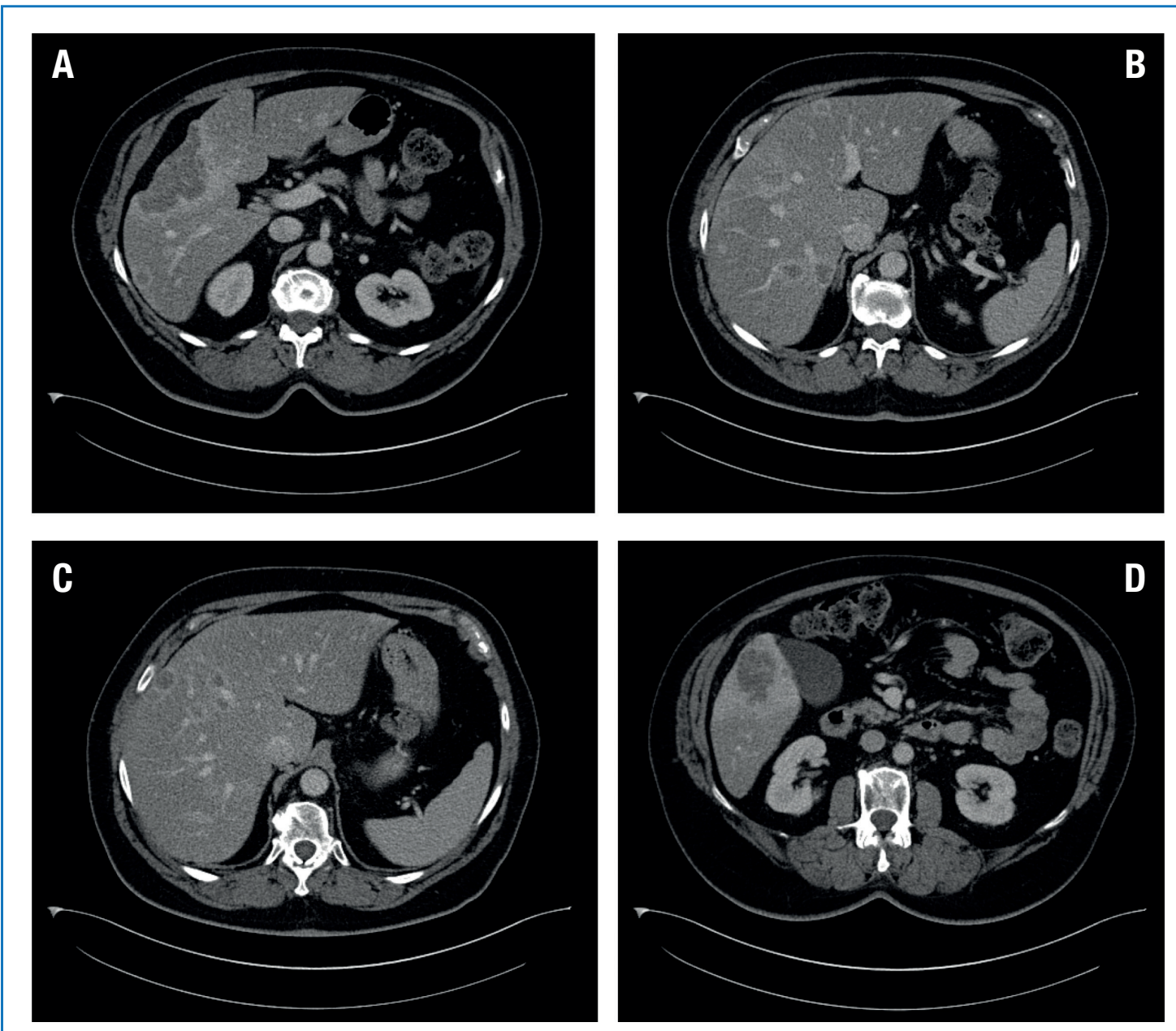

Figura 1. Tomografia computerizzata (TC) di gennaio 2014. (A) multipli noduli epatici di cui il maggiore di dimensione $107 \times 93 \times 50$ mm al V segmento epatico; (B) due nodulazioni strettamente adiacenti fra loro in corrispondenza dei segmenti VII e VIII; (C) quattro nodulazioni ravvicinate al IV segmento; (D) una lesione a margini irregolari al V segmento.

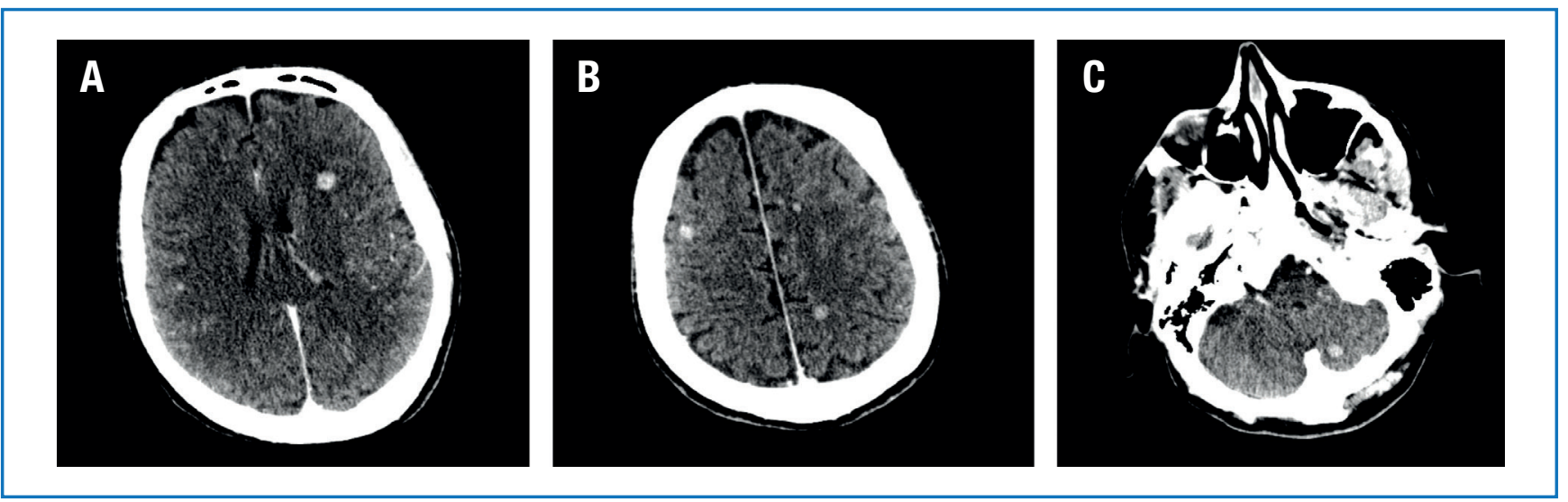

Figura 2. TC total body di giugno 2014 che evidenzia varie lesioni in sedi cerebrali diverse: (A) corona radiata (B) sede frontale sottocorticale e (C) cerebellare sinistra. 

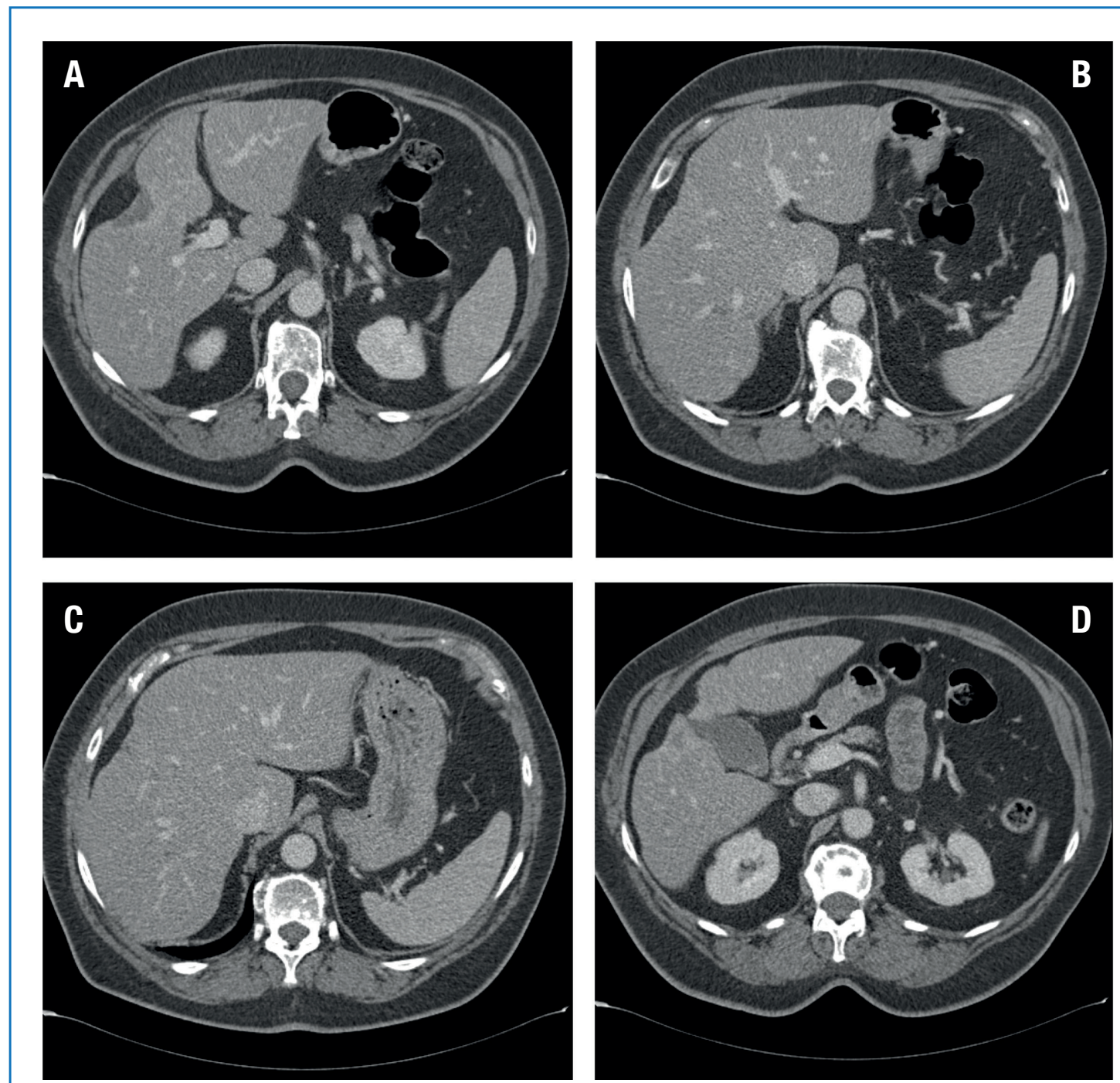

Figura 3. Tomografia computerizzata (TC) di rivalutazione a febbraio 2015, con evidenza di risposta delle lesioni target epatiche.

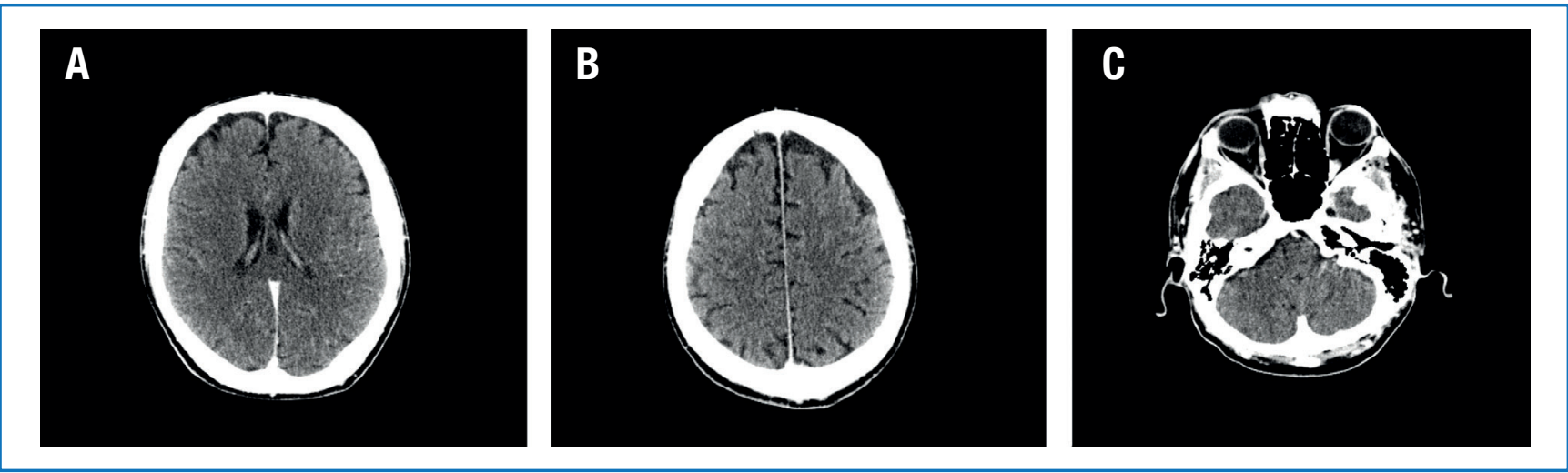

Figura 4. Tomografia computerizzata (TC) di rivalutazione a febbraio 2015, con evidenza di risposta delle lesioni encefaliche. 


\section{Considerazioni cliniche}

Le donne con carcinoma della mammella caratterizzato da sovra-espressione di HER2 presentano un maggiore rischio di progressione di malattia e di mortalità rispetto alle donne con carcinoma della mammella luminale [1]. Nuove strategie terapeutiche, come trastuzumab, pertuzumab, TDM1 e lapatinib, hanno radicalmente modificato la sopravvivenza di queste pazienti [2-4]. Il rischio che una donna con carcinoma della mammella sviluppi metastasi cerebrali è del 20-30\% [5], che aumenta fino al 40\% se la malattia presenta over-espressione del recettore HER2 [6]

Lapatinib è in grado di oltrepassare la barriera ematoencefalica e si è dimostrato efficace nel trattamento delle metastasi cerebrali $[7,8]$. La concentrazione di lapatinib è significativamente più alta nelle metastasi cerebrali rispetto ad altri sedi extracraniche e inoltre il farmaco non si concentra nel tessuto cerebrale sano [9]. Lapatinib ha anche un'azione multi-target inibendo in modo competitivo e reversibile i domini intracellulari dei recettori HER1 e HER2 $[10,11]$; a ciò si aggiunge il fatto che, diversamente da trastuzumab, è in grado di inibire anche la forma tronca p95Her-2, priva di dominio extracellulare del recettore HER2 [12].

È ormai assodato che la terapia delle metastasi cerebrali necessita di una gestione non solo medica, ma anche chirurgica e/o radioterapica (whole-brain $\mathrm{o}$ radioterapia stereotassica in base al numero e alle dimensioni delle lesioni encefaliche): diversi studi hanno dimostrato come la combinazione di trattamenti locoregionali (chirurgia o radioterapia) farmaci chemioterapici e terapie target anti-HER2 prolunghi in modo significativo la sopravvivenza di queste pazienti [13].

Per quanto concerne la combinazione con un farmaco chemioterapico, l'associazione lapatinib con capecitabina si è dimostrata superiore rispetto alla sola capecitabina, con un aumento significativo dell'intervallo libero da progressione ( 8,4 vs 4,4 mesi, rispettivamente) [13]. Entrambi questi farmaci hanno la capacità di concentrarsi in modo altamente selettivo nelle metastasi cerebrali da carcinoma della mammella HER2-positivo [14]; inoltre lapatinib, riducendo l'espressione della timidilato sintetasi, enzima inibito da capecitabina, potenzia l'azione antineoplastica di quest'ultima [15]

Lo studio di fase II LANDSCAPE ha dimostrato che la combinazione lapatinib-capecitabina è un trattamento di I linea efficace sulle lesioni encefaliche non pretrattate [16]. La metanalisi di Petrelli et al. [17], condotta su 800 donne con carcinoma mammario HER2-positivo con metastasi encefaliche e arruolate in 12 differenti studi, ha dimostrato che questa combinazione si associa a una percentuale di risposta globale del 29\%, a una sopravvivenza mediana libera da progressione di 4 mesi e a una sopravvivenza mediana globale di 11 mesi.

Alla luce dei dati sopracitati e in considerazione della progressione encefalica dopo trattamento standard di I linea (taxano-pertuzumab-trastuzumab), si è optato per un trattamento di II linea con capecitabina-lapatinib ottenendo un lungo controllo di malattia. 


\section{Disclosure}

Questa pubblicazione è stata resa possibile grazie a un grant non condizionante di Istituto Gentili.

I dott. V. Magri, S. Scagnoli, G. Piesco e G. Pomati dichiarano di non avere conflitti di interesse in relazione all'articolo pubblicato.

\section{Bibliografia}

1. Parkin DM, Bray F, Ferlay J, et al. Global cancer statistics, 2002. CA Cancer J Clin. 2005;55:74-108.

2. Slamon DJ, Leyland-Jones B, Shak S, et al. Use of chemotherapy plus a monoclonal antibody against HER2 for metastatic breast cancer that overexpresses HER2. N Engl J Med. 2001;344:783-92.

3. Swain SM, Kim SB, Cotes J, et al. Pertuzumab, trastuzumab and docetaxelforHER2-positivemetastaticbreastcancer(CLEOPATRA study): overall survival results from a randomised, doubleblind, placebo controlled, phase 3 study. Lancet Oncol. 2013; 14:461-71.

4. Verna S, Miles D, Gianni L, et al. EMILIA study group. Trastuzumab emtansine for HER2-positive advanced breast cancer. N Engl J Med. 2012;367:1783-91.

5. Tabouret E, Chinot O, Metellus $\mathrm{P}$, et al. Recent trends in epidemiology of brain metastases: an overview. Anticancer Res. 2012;32:4655-62.

6. Bendell J, Domchek S, Burstein H, et al. Central nervous system metastases in women who receive trastuzumab-based therapy for metastatic breast carcinoma. Cancer. 2003;97:2972-77.

7. Gril B, Palmieri D, Bronder JL, et al. Effect of lapatinib on the outgrowth of metastatic breast cancer cells to the brain. J Natl Cancer Inst. 2008;100:1092-103

8. Saleem A, Searle GE, Kenny LM, et al. Lapatinib access into normal brain and brain metastases in patients with Her-2 overexpressing breast cancer. EJNMMI Res. 2017;7:76

9. Rusnak DW, Lackey K, Affleck K, et al. The effects of the novel, reversible epidermal growth factor receptor/ErbB-2 tyrosine kinase inhibitor, GW2016, on the growth of human normal and tumor-derived cell lines in vitro and in vivo. Mol Cancer Ther. 2001; 1:85-94

10. Spector NL, Xia W, Burris H III, et al. Study of the biological effects of lapatinib, a reversible inhibitor of ErbB1 and ErbB2 tyrosine kinases, on tumor growth and survival pathways in patients with advanced malignancies. J Clin Oncol. 2005;23:2502-12.

11. Ahn ER, Vogel CL. Dual HER2-targeted approaches in HER2 positive breast cancer. Breast Cancer Res Treat. 2012;131:371-83.

12. Brufsky AM, Mayer M, Rugo HS, et al. Central nervous system metastases in patients with HER2 positive metastatic breast cancer: incidence, treatment, and survival in patients from registHER. Clin Cancer Res. 2011;17(14):4834-43

13. Geyer CE, Forster J, Lindquist D, et al. Lapatinib plus Capecitabina for HER2-positive advanced breast cancer. N Engl J Med. 2006;355:2733-43..

14. Morikawa A, Peereboom DM, Thorsheim HR, et al. Capecitabine and lapatinib uptake in surgical resected brain metastases from metastatic breast cancer patients: a prospective study. Neuro Oncol. 2015;17:289-95.

15. Chefrour M1, Milano G, Formento P, et al. Positive interaction between lapatinib and capecitabine in human breast cancer models: study of molecular determinants. Fundam Clin Pharmacol. 2012;26(4):530-7.

16. Bachelot $T$, Romieu $G$, Campone $M$, et al. Lapatinib plus capecitabine in patients with previously untreated brain metastases from HER2-positive metastatic breast cancer (LANDSCAPE): a single-group phase 2 study. Lancet Oncol. 2013;14:64-71.

17. Petrelli F, Ghidini M, Lonati V, et al. The efficacy of lapatinib and capecitabine in HER-2 positive breast cancer with brain metastases: A systematic review and pooled analysis. Eur J Cancer. 2017;84:141-8. 